\title{
On the leximin and utilitarian overtaking criteria with extended anonymity*
}

\author{
Kohei Kamaga $^{\dagger} \quad$ Takashi Kojima ${ }^{\ddagger}$ \\ First version, December 18, 2007; \\ Current version, August 21, 2008
}

\begin{abstract}
This paper studies the extensions of the infinte-horizon variants of the leximin principle and utilitarianism on the set of infinite utility streams. We especially examine those extensions which satisfy the axiom of Preference-continuity (or Consistency) and the extended anonymity axiom called $\mathcal{Q}$-Anonymity. We formulate new extended leximin and utilitarian social welfare relations (SWRs), called $\mathcal{Q}$-W-leximin SWR and $\mathcal{Q}$-overtaking criterion respectively, and show that Weak Preference-continuity (or Weak Consistency) and $\mathcal{Q}$-Anonymity together with Strong Pareto and Hammond Equity (resp. Partial Unit Comparability) characterize all SWRs that include the $\mathcal{Q}$-W-leximin SWR (resp. the $\mathcal{Q}$-overtaking criterion) as a subrelation. We also show that there exists no SWR satisfying Strong Pareto, Strong Preference-continuity (or Strong Consistency) and $\mathcal{Q}$-Anonymity.
\end{abstract}

Keywords $\mathcal{Q}$-Anonymity; Preference-continuity; Consistency; Leximin; Utilitarianism; Overtaking criterion

JEL Classification Numbers D63; D70

\footnotetext{
*The earlier draft of this paper was entitled " $\mathcal{Q}$-Anonymity and preference continuity" or "On the extensions of the infinite-horizon leximin and the overtaking criteria." We are grateful for helpful comments and suggestions from Marc Fleurbaey, Yoshio Kamijo, Shigeaki Mizutani, Tetsuro Okazaki, Hiroo Sasaki, and Koichi Suga. Remaining errors are our own.

${ }^{\dagger}$ Graduate School of Economics, Waseda University, 1-6-1, Nishi-waseda, Shinjuku-ku, Tokyo 169-8050, Japan (E-mail: k-kmg@ruri.waseda.jp)

${ }^{\ddagger}$ Graduate School of Economics, Waseda University, 1-6-1, Nishi-waseda, Shinjuku-ku, Tokyo 169-8050, Japan (E-mail: tkojima@ruri.waseda.jp)
} 


\section{Introduction}

In evaluating infinite-horizon utility streams, Strong Pareto and Finite Anonymity are the most common principles employed in the literature. The former is the requirement of efficiency (or sensitivity) and the latter is that of impartiality among generations. These basic principles lead us to the infinite-horizon variant of the Suppes-Sen grading principle (Svensson 1980; Asheim et al. 2001). ${ }^{1}$ The infinite-horizon Suppes-Sen grading principle compares two utility streams only by the Pareto dominance after a transformation by a suitable finite permutation. Hence, what the Suppes-Sen grading principle by itself asserts on our evaluation is quite weak and, consequently, many utility streams will be declared to be non-comparable.

Further comparability beyond the Suppes-Sen grading principle has been pursued along two rival principles of justice: Rawlsian lexicographic maximin principle and utilitarianism. Basu and Mitra (2007) formulate and characterize the infinite-horizon variant of utilitarianism (henceforth, utilitarian social welfare relation (SWR)). The utilitarian SWR applies the well-established finite-horizon utilitarian ordering to the first $n$ generations' utilities and the Pareto principle to the utilities of subsequent future generations. ${ }^{2}$ In a similar manner, the infinite-horizon variant of the leximin principle, called leximin SWR, is formulated and characterized by Bossert et al. (2007) with the finite-horizon leximin ordering and the Pareto principle. These SWRs are characterized by adding one auxiliary axiom to Strong Pareto and Finite Anonymity: Partial Unit Comparability in the case of the utilitarian SWR and Hammond Equity in the case of the leximin SWR. Both two exhibit higher level of comparability than the Suppes-Sen grading principle. However, since the Pareto principle, applied to future generations' utilities, is an incomplete quasi-ordering, we cannot compare many utility streams that involves a conflict among infinitely many generations.

To give a resolution to conflicts involving infinitely many generations, two different kinds of extensions of the leximin and utilitarian SWRs have been proposed in the literature. $^{3}$ The first one is the extensions considered by Asheim and Tungodden (2004) and Basu and Mitra (2007). They employ an additional axiom called Preference-continuity or Consistency respectively. Preference-continuity and Consistency formalize a quite similar requirement. Both two basically assert that our comparisons of infinite-horizon utility streams should be consistent with an infinite number of comparisons of finitehorizon truncated paths. Adding Weak (resp. Strong) Preference-continuity, Asheim

\footnotetext{
${ }^{1}$ The Suppes-Sen grading principle is originally formulated in a finite population setting. See Suppes (1966) and Sen (1970).

${ }^{2}$ This type of SWR is generically referred to as simplified criterion in d'Aspremont (2007).

${ }^{3}$ The extensions we introduce here do not exhaust all the existing ones. See, for example, Fleurbaey and Michel (2003). Focusing on the notions of time-invariance and stationarity, Asheim et al. (2008) recently propose the generalized time-invariant overtaking criterion. The leximin and utilitarian versions of their extended criterion exhibit higher level of comparability than the leximin and utilitarian SWRs respectively.
} 


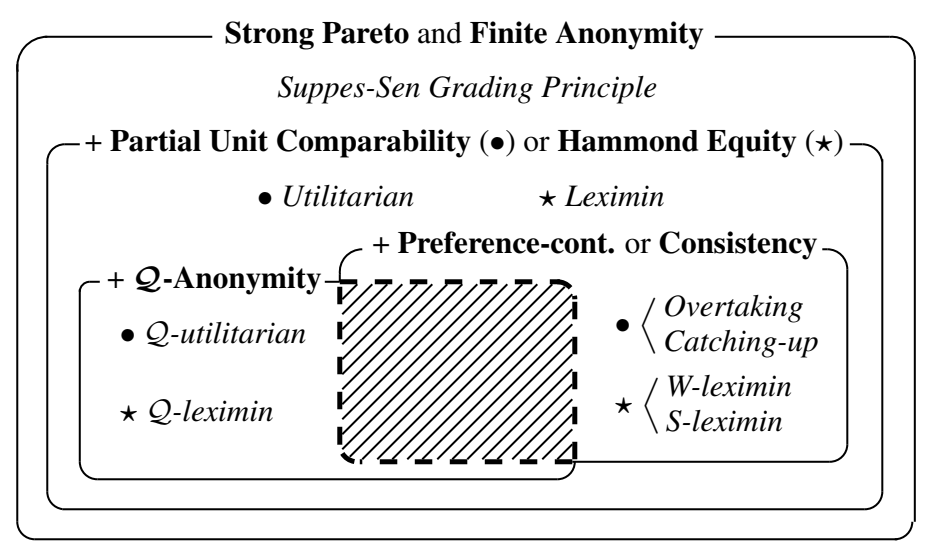

Figure 1: Characterizations of classes of SWRs and their least elements

and Tungodden (2004) characterize the extended leximin SWR called W-leximin (resp. S-leximin) SWR and the well-known extended utilitarian SWR called overtaking (resp. catching-up) criterion. Basu and Mitra (2007) also characterize the overtaking and catching-up criteria with two versions of consistency. In these extended criteria, the notion of Preference-continuity or Consistency is crystallized as an infinite number of application of the finite-horizon leximin and utilitarian orderings respectively. Therefore, these SWRs make further comparisons beyond the limits of the leximin and utilitarian SWRs and will provide more selected maximal paths.

The other type of extension is proposed by Banerjee (2006) and also analyzed in Kamaga and Kojima (2008). ${ }^{4}$ They strengthen Finite Anonymity to the extended anonymity called $\mathcal{Q}$-Anonymity. $\mathcal{Q}$-Anonymity is first introduced by Lauwers (1997b) under the name Fixed Step Anonymity and is defined by a certain restricted class of infinite permutations that includes all finite permutations. ${ }^{5}$ The most common example that illustrates the difference between Finite Anonymity and $\mathcal{Q}$-Anonymity is the streams $\boldsymbol{x}=(1,0,1,0, \ldots)$ and $\boldsymbol{y}=(0,1,0,1, \ldots)$. While Finite Anonymity cannot provide a definite ranking of $\boldsymbol{x}$ and $\boldsymbol{y}, \mathcal{Q}$-Anonymity declares them to be indifferent. Banerjee (2006) characterizes the $\mathcal{Q}$-utilitarian $S W R$ with $\mathcal{Q}$-Anonymity. Its leximin counterpart, called $\mathcal{Q}$-leximin $S W R$, is characterized by Kamaga and Kojima (2008). While the leximin and utilitarian SWRs declare the streams $\boldsymbol{x}$ and $\boldsymbol{y}$ to be noncomparable, the $\mathcal{Q}$-leximin and $\mathcal{Q}$-utilitarian SWR ensure social indifference between them, a quite intuitive evaluation. The existing characterizations we mentioned here are summarized in Figure 1.

The purpose of this paper is to formulate and characterize new extended leximin

\footnotetext{
${ }^{4}$ See also Mitra and Basu (2007).

${ }^{5}$ See also Fleurbaey and Michel (2003) and Sakai (2008), where other relevant anonymity axioms are discussed in a comprehensive manner.
} 
and utilitarian SWRs that satisfy both Preference-continuity or Consistency and $\mathcal{Q}$ Anonymity, i.e. those extended criteria which incorporate both merits of the extensions by Asheim and Tungodden (2004) and Basu and Mitra (2007) and by Banerjee (2006) and Kamaga and Kojima (2008). In Figure 1, the shaded area corresponds to the class we are interested in. It is well-known that the catching-up criterion (the extended utilitarian SWR corresponding to the case of the strong versions of preference-continuity and consistency) violates $\mathcal{Q}$-Anonymity. ${ }^{6}$ Our first result shows that this impossibility can be ascribed to the incompatibility of Strong Preference-continuity (or Strong Consistency) and $\mathcal{Q}$-Anonymity in a strongly Paretian SWR. This impossibility result tells that the shaded area in Figure 1 is empty in the case of Strong Preference-continuity or Strong Consistency and our choice of $\mathcal{Q}$-Anonymity or Strong Preference-continuity (or Strong Consistency) is a branching point in exploring the SWRs that make further comparisons beyond the Suppes-Sen grading principle.

In contrast to the cases of Strong Preference-continuity and Strong Consistency, our second result give an affirmative answer to the possibility of $\mathcal{Q}$-anonymous and preference-continuous (or consistent) extensions of the leximin and utilitarian SWRs. We formulate $\mathcal{Q}$-anonymous extensions of the W-leximin SWR and the overtaking criterion, called $\mathcal{Q}$-W-leximin $S W R$ and $\mathcal{Q}$-overtaking criterion respectively. Then, we show that both two extended criteria are well-defined as a SWR on infinite utility streams and that Weak Preference-continuity (or Weak Consistency) and $\mathcal{Q}$-Anonymity together with Strong Pareto, Finite Anonymity and Hammond Equity characterize all SWRs that include the $\mathcal{Q}$-W-leximin SWR as a subrelation. We also establish the characterization of the $\mathcal{Q}$-overtaking criterion by replacing with Hammond Equity with Partial Unit Comparability.

The rest of the paper is organized as follows. Section 2 presents notation and definitions. The axioms we impose on SWRs are also introduced. Section 3 provides the results obtained in this paper. In Section 4, we compare our new SWRs with some well-established ones. Section 5 concludes with some remarks.

\section{Preliminary}

\subsection{Notation and definitions}

Let $\mathbb{R}$ (resp. $\mathbb{R}_{++}$) be the set of all (resp. all positive) real numbers and $\mathbb{N}$ the set of all positive integers. Let $X=\mathbb{R}^{\mathbb{N}}$ be the set of all utility streams $\boldsymbol{x}=\left(x_{1}, x_{2}, \ldots\right)$. For all $i \in \mathbb{N}, x_{i}$ is interpreted as the utility level of the $i$ th generation. For all $\boldsymbol{x} \in X$ and all $n \in \mathbb{N}$, we write $\boldsymbol{x}^{-n}=\left(x_{1}, \ldots, x_{n}\right)$ and $\boldsymbol{x}^{+n}=\left(x_{n+1}, x_{n+2}, \ldots\right)$. For all

\footnotetext{
${ }^{6}$ On this, see Example 1 in Banerjee (2006).
} 
$\boldsymbol{x} \in X$ and all $n \in \mathbb{N},\left(x_{(1)}^{-n}, \ldots, x_{(n)}^{-n}\right)$ denotes a rank-ordered permutation of $\boldsymbol{x}^{-n}$ such that $x_{(1)}^{-n} \leq \cdots \leq x_{(n)}^{-n}$, ties being broken arbitrarily.

A SWR, denoted by $\succsim$, is a reflexive and transitive binary relation on $X$, i.e. a quasi-ordering. ${ }^{7}$ An asymmetric part of $\succsim$ is denoted by $\succ$ and a symmetric part by $\sim$, i.e. $\boldsymbol{x} \succ \boldsymbol{y}$ if and only if $\boldsymbol{x} \succsim \boldsymbol{y}$ holds but $\boldsymbol{y} \succsim \boldsymbol{x}$ does not, and $\boldsymbol{x} \sim \boldsymbol{y}$ if and only if $\boldsymbol{x} \succsim \boldsymbol{y}$ and $\boldsymbol{y} \succsim \boldsymbol{x}$. A SWR $\succsim_{A}$ is said to be a subrelation of a SWR $\succsim_{B}$ if, for all $\boldsymbol{x}, \boldsymbol{y} \in X$, (i) $\boldsymbol{x} \sim_{A} \boldsymbol{y}$ implies $\boldsymbol{x} \sim_{B} \boldsymbol{y}$ and (ii) $\boldsymbol{x} \succ_{A} \boldsymbol{y}$ implies $\boldsymbol{x} \succ_{B} \boldsymbol{y}$.

Following Mitra and Basu (2007) and Banerjee (2006), we represent any permutation on the set $\mathbb{N}$ by a permutation matrix. A permutation matrix is an infinite matrix $\boldsymbol{P}=\left(p_{i j}\right)_{i, j \in \mathbb{N}}$ satisfying the following properties:

(i) for each $i \in \mathbb{N}$, there exists $j(i) \in \mathbb{N}$ such that $p_{i j(i)}=1$ and $p_{i j}=0$ for all $j \neq j(i)$;

(ii) for each $j \in \mathbb{N}$, there exists $i(j) \in \mathbb{N}$ such that $p_{i(j) j}=1$ and $p_{i j}=0$ for all $i \neq i(j)$.

Let $\mathcal{P}$ be the set of all permutation matrices. Note that, for all $\boldsymbol{x} \in X$ and all $\boldsymbol{P} \in \mathcal{P}$, the product $\boldsymbol{P} \boldsymbol{x}=\left(P x_{1}, P x_{2}, \ldots\right)$ belongs to $X$, where $P x_{i}=\sum_{k \in \mathbb{N}} p_{i k} x_{k}$ for all $i \in \mathbb{N}$. For any $\boldsymbol{P} \in \mathcal{P}$, let $\boldsymbol{P}^{\prime}$ be the inverse of $\boldsymbol{P}$ satisfying $\boldsymbol{P}^{\prime} \boldsymbol{P}=\boldsymbol{P} \boldsymbol{P}^{\prime}=\boldsymbol{I}$, where $\boldsymbol{I}$ is the infinite identity matrix. ${ }^{8}$ For all $\boldsymbol{P}=\left(p_{i j}\right)_{i, j \in \mathbb{N}} \in \mathcal{P}$ and all $n \in \mathbb{N}$, let $\boldsymbol{P}(n)$ denote the $n \times n$ matrix $\left(p_{i j}\right)_{i, j \in\{1, \ldots, n\}}$. A matrix $\boldsymbol{P}=\left(p_{i j}\right)_{i, j \in \mathbb{N}} \in \mathcal{P}$ is a finite permutation matrix if there exists $n \in \mathbb{N}$ such that $p_{i i}=1$ for all $i>n$. Let $\mathcal{F}$ be the set of all finite permutation matrices.

Let $e^{i}$ be the stream in $X$ with 1 in the $i$ th place and 0 elsewhere, i.e. the $i$ th unit vector in $X$. A permutation $\boldsymbol{P} \in \mathcal{P}$ is said to be cyclic if, for any $i \in \mathbb{N}$, there exists $k(i) \in \mathbb{N}$ such that $\boldsymbol{P}^{k(i)} \boldsymbol{e}^{i}=\boldsymbol{e}^{i}$, where $\boldsymbol{P}^{k(i)}$ denotes the $k(i)$ times iterated multiplication of $\boldsymbol{P}$. While $\mathcal{P}$ and $\mathcal{F}$ define a group with respect to the matrix multiplication, a special class of cyclic permutations does not (e.g. the class of all cyclic permutations). ${ }^{9}$ As shown by Mitra and Basu (2007), any (and only) group(s) of cyclic permutations can define the anonymity axiom consistent with a strongly Paretian SWR. As in Banerjee (2006), we focus on the following group $\mathcal{Q}$ of cyclic permutations:

$$
\mathcal{Q}=\left\{\boldsymbol{P} \in \mathcal{P}: \begin{array}{l}
\text { there exists } k \in \mathbb{N} \text { such that, for each } n \in \mathbb{N}, \\
\boldsymbol{P}(n k) \text { is a finite-dimensional permutation matrix }
\end{array}\right\} .
$$

\footnotetext{
${ }^{7}$ A binary relation $\succsim$ on $X$ is (i) reflexive if, for all $\boldsymbol{x} \in X, \boldsymbol{x} \succsim \boldsymbol{x}$, and (ii) transitive if, for all $\boldsymbol{x}, \boldsymbol{y}, \boldsymbol{z} \in X, \boldsymbol{x} \succsim \boldsymbol{z}$ holds whenever $\boldsymbol{x} \succsim \boldsymbol{y}$ and $\boldsymbol{y} \succsim \boldsymbol{z}$.

${ }^{8}$ For any $\boldsymbol{P}, \widetilde{Q} \in \mathcal{P}$, the product $\boldsymbol{P} \boldsymbol{Q}$ is defined by $\left(r_{i j}\right)_{i, j \in \mathbb{N}}$ with $r_{i j}=\sum_{k \in \mathbb{N}} p_{i k} q_{k j}$.

${ }^{9}$ For any $\mathcal{Q} \subseteq \mathcal{P}, \mathcal{Q}$ is said to define a group w.r.t. the matrix multiplication if (i) for all $\boldsymbol{P}, \boldsymbol{Q} \in \mathcal{Q}$, $\boldsymbol{P Q} \in \mathcal{Q}$, (ii) there exists $\boldsymbol{I} \in \mathcal{Q}$ such that for all $\boldsymbol{P} \in \mathcal{Q}, \boldsymbol{I} \boldsymbol{P}=\boldsymbol{P I}=\boldsymbol{P}$, (iii) for all $\boldsymbol{P} \in \mathcal{Q}$, there exists $\boldsymbol{P}^{\prime} \in \mathcal{Q}$ such that $\boldsymbol{P}^{\prime} \boldsymbol{P}=\boldsymbol{P} \boldsymbol{P}^{\prime}=\boldsymbol{I}$, and (iv) for all $\boldsymbol{P}, \boldsymbol{Q}, \boldsymbol{R} \in \mathcal{Q},(\boldsymbol{P Q}) \boldsymbol{R}=\boldsymbol{P}(\boldsymbol{Q R})$.
} 
The class $\mathcal{Q}$ is exactly the set of all fixed step permutations. The class of fixed step permutations is first introduced by Lauwers (1997b). ${ }^{10}$

Negation of a statement is indicated by the logic symbol $\neg$. Our notation for vector inequalities on $X$ is as follows: for all $\boldsymbol{x}, \boldsymbol{y} \in X, \boldsymbol{x} \geqslant \boldsymbol{y}$ if $x_{i} \geq y_{i}$ for all $i \in \mathbb{N}$, and $\boldsymbol{x}>\boldsymbol{y}$ if $\boldsymbol{x} \geqslant \boldsymbol{y}$ and $\boldsymbol{x} \neq \boldsymbol{y}$.

\subsection{Axioms}

\subsubsection{Basic axioms}

We introduce some basic axioms that provide axiomatic foundations of the infinitehorizon variants of the leximin and utilitarian orderings.

We begin with two guiding principles of sensitivity and impartiality.

Strong Pareto (SP) For all $\boldsymbol{x}, \boldsymbol{y} \in X$, if $\boldsymbol{x}>\boldsymbol{y}$, then $\boldsymbol{x} \succ \boldsymbol{y}$.

$\mathcal{F}$-Anonymity (FA) For all $\boldsymbol{x} \in X$ and all $\boldsymbol{P} \in \mathcal{F}, \boldsymbol{P} \boldsymbol{x} \sim \boldsymbol{x}$.

FA is also called Finite (or Weak) Anonymity.

The next one is an infinite-horizon variant of the well-known consequentialist equity axiom introduced by Hammond (1976).

Hammond equity (HE) For all $\boldsymbol{x}, \boldsymbol{y} \in X$ and all $i, j \in \mathbb{N}$, if $y_{i}<x_{i}<x_{j}<y_{j}$ and for all $k \in \mathbb{N} \backslash\{i, j\}, x_{k}=y_{k}$, then $\boldsymbol{x} \succsim \boldsymbol{y}$.

HE asserts that an order-preserving change which diminishes inequality of utilities between conflicting two generations is socially preferable. The leximin SWR is characterized by SP, FA and HE (Bossert et al. 2007). The definition of the leximin SWR is available in Sect. 4.

We move to the following two informational invariance axioms.

Partial unit comparability (PUC) For all $\boldsymbol{x}, \boldsymbol{y} \in X$, all $\boldsymbol{a} \in \mathbb{R}^{\mathbb{N}}$ and all $n \in \mathbb{N}$, if $\boldsymbol{x}^{+n}=\boldsymbol{y}^{+n}$ and $\boldsymbol{x} \succsim \boldsymbol{y}$, then $\boldsymbol{x}+\boldsymbol{a} \succsim \boldsymbol{y}+\boldsymbol{a}$.

2-Generation unit comparability (2UC) For all $\boldsymbol{x}, \boldsymbol{y} \in X$, all $i, j \in \mathbb{N}$, and all $\boldsymbol{a} \in \mathbb{R}^{\mathbb{N}}$ if, for all $k \neq i, j, a_{k}=0$ and $\boldsymbol{x} \succsim \boldsymbol{y}$, then $\boldsymbol{x}+\boldsymbol{a} \succsim \boldsymbol{y}+\boldsymbol{a}$.

PUC is employed in Basu and Mitra (2007) and 2UC in Asheim and Tungodden (2004). Although the definitions of them are slightly different, both two basically assert that utility differences of generations are comparable but utility levels are not. ${ }^{11}$

\footnotetext{
${ }^{10}$ Lauwers (2006) shows that the class $\mathcal{Q}$ of fixed step permutations is not a maximal group of cyclic permutations.

${ }^{11}$ Since $\mathbf{H E}$ assumes at least ordinally measurable and level comparable utilities, it is incompatible with 2UC and PUC. For the detailed explanation of informational invariance axioms, we refer the reader to Bossert and Weymark (2004) and d'Aspremont and Gevers (2002).
} 
PUC (or 2UC) together with SP and FA characterizes the utilitarian SWR (Basu and Mitra 2007). ${ }^{12}$ For the formal definition of the utilitarian SWR, see Sect. 4.

\subsubsection{Additional axioms}

We now introduce additional axioms that are used to characterize the extended leximin and utilitarian SWRs.

We begin with the axioms employed by Asheim and Tungodden (2004) and Basu and Mitra (2007). Asheim and Tungodden (2004) consider two versions of preferencecontinuity axioms.

Weak preference-continuity (WPC) For all $\boldsymbol{x}, \boldsymbol{y} \in X$, if there exists $\bar{n} \in \mathbb{N}$ such that for all $n \geq \bar{n},\left(\boldsymbol{x}^{-n}, \boldsymbol{y}^{+n}\right) \succ \boldsymbol{y}$, then $\boldsymbol{x} \succ \boldsymbol{y}$.

Strong preference-continuity (SPC) For all $\boldsymbol{x}, \boldsymbol{y} \in X$, if there exists $\bar{n} \in \mathbb{N}$ such that for all $n \geq \bar{n},\left(\boldsymbol{x}^{-n}, \boldsymbol{y}^{+n}\right) \succsim \boldsymbol{y}$, and for all $\bar{n} \in \mathbb{N}$, there exists $n \geq \bar{n}$ such that $\left(\boldsymbol{x}^{-n}, \boldsymbol{y}^{+n}\right) \succ \boldsymbol{y}$, then $\boldsymbol{x} \succ \boldsymbol{y}$.

Basu and Mitra (2007) employ the following consistency axioms.

Weak consistency (WC) For all $\boldsymbol{x}, \boldsymbol{y} \in X$, (i) if there exists $\bar{n} \in \mathbb{N}$ such that for all $n \geq \bar{n},\left(\boldsymbol{x}^{-n}, 0,0, \ldots\right) \succ\left(\boldsymbol{y}^{-n}, 0,0, \ldots\right)$, then $\boldsymbol{x} \succ \boldsymbol{y}$; (ii) if there exists $\bar{n} \in \mathbb{N}$ such that for all $n \geq \bar{n},\left(\boldsymbol{x}^{-n}, 0,0, \ldots\right) \sim\left(\boldsymbol{y}^{-n}, 0,0, \ldots\right)$, then $\boldsymbol{x} \sim \boldsymbol{y}$.

Strong consistency (SC) For all $\boldsymbol{x}, \boldsymbol{y} \in X$, (i) if there exists $\bar{n} \in \mathbb{N}$ such that for all $n \geq \bar{n},\left(\boldsymbol{x}^{-n}, 0,0, \ldots\right) \succsim\left(\boldsymbol{y}^{-n}, 0,0, \ldots\right)$, then $\boldsymbol{x} \succsim \boldsymbol{y}$; (ii) if there exists $\bar{n} \in \mathbb{N}$ such that for all $n \geq \bar{n},\left(\boldsymbol{x}^{-n}, 0,0, \ldots\right) \succsim\left(\boldsymbol{y}^{-n}, 0,0, \ldots\right)$ and for all $\bar{n} \in \mathbb{N}$, there exists $n \geq \bar{n}$ such that $\left(\boldsymbol{x}^{-n}, 0,0, \ldots\right) \succ\left(\boldsymbol{y}^{-n}, 0,0, \ldots\right)$, then $\boldsymbol{x} \succ \boldsymbol{y}$.

Both WPC and WC (and also SPC and SC) are defined similarly in spirit to Axiom 3 in Brock (1970) and basically assert that our comparison of infinite-horizon utility streams should be consistent with the comparisons of their finite-horizon truncated paths if the length of truncations are large enough. Indeed, these axioms are equivalent in the class of SWRs that include the leximin or utilitarian SWR as a subrelation in both cases of strong and weak versions of them. ${ }^{13}$

Next, we introduce the axiom employed in Banerjee (2006) and Kamaga and Kojima (2008). Instead of FA, they impose the following stronger anonymity axiom.

$\mathcal{Q}$-Anonymity (QA) For all $\boldsymbol{x} \in X$ and all $\boldsymbol{P} \in \mathcal{Q}, \boldsymbol{P} \boldsymbol{x} \sim \boldsymbol{x}$.

\footnotetext{
${ }^{12}$ For the case of $\mathbf{2 U C}$, see the argument in the proof of Proposition 4 in Asheim and Tungodden (2004).

${ }^{13}$ It should be noted that $\mathbf{S P}$ and the following independence implied by any of the utilitarian and leximin SWRs suffice for this equivalence: for all $\boldsymbol{x}, \boldsymbol{y}, \boldsymbol{x}^{\prime}, \boldsymbol{y}^{\prime} \in X$, if there exists $n \in \mathbb{N}$ such that $\boldsymbol{x}^{-n}=\boldsymbol{x}^{\prime-n}$ and $\boldsymbol{y}^{-n}=\boldsymbol{y}^{\prime-n}$, and $\boldsymbol{x}^{+n}=\boldsymbol{y}^{+n}$ and $\boldsymbol{x}^{++n}=\boldsymbol{y}^{\prime+n}$, then $\boldsymbol{x} \succsim \boldsymbol{y}$ iff $\boldsymbol{x}^{\prime} \succsim \boldsymbol{y}^{\prime}$.
} 
QA is also called Fixed Step Anonymity (Lauwers 1997b). ${ }^{14}$

For each of the additional axioms, the characterizations of the extended leximin and utilitarian SWRs are already established: W-leximin SWR and overtaking criterion with WPC or WC (Asheim and Tungodden 2004; Basu and Mitra 2007); S-leximin SWR and catching-up criterion with SPC or SC (Asheim and Tungodden 2004; Basu and Mitra 2007); and Q-utilitarian and Q-leximin SWRs with QA (Banerjee 2006; Kamaga and Kojima 2008). The definitions of these SWRs are available in Sections 3 and 4 (and Footnote 20).

\section{Further extensions and characterizations}

The principal task of this paper is to establish characterizations of the extended leximin and utilitarian SWRs that satisfy both of the two different kinds of additional axioms, one of the four axioms of preference-continuity or consistency and QA, i.e. the characterizations of those extended SWRs which incorporate the merits of the extensions by Asheim and Tungodden (2004) and Basu and Mitra (2007) and by Banerjee (2006) and Kamaga and Kojima (2008). Since, as we noted earlier, it is impossible to formulate the extension of the utilitarian SWR (and the leximin SWR) satisfying QA and SPC or SC. our interest lies particularly on the possibility of the extended leximin and utilitarian SWRs that satisfy both QA and WPC or WC.

Before proceeding to the main issue, we show that the impossibility for cases of the stronger versions of preference-continuity and consistency is ascribed to the incompatibility between QA and SPC or SC in a strongly Paretian SWR.

Proposition 1. (i) There exists no $S W R \succsim$ satisfying $\boldsymbol{S P}, \boldsymbol{Q A}$, and $\boldsymbol{S P C}$. (ii) There exists no $S W R \succsim$ satisfying $\boldsymbol{S P}, \boldsymbol{Q A}$, and $\boldsymbol{S C}$.

Proof. See Appendix.

The trade-off between efficiency formalized as Paretian axioms and impartiality done by anonymity axioms has been intensively analyzed in the literature. As we noted in the preceding section, $\mathbf{Q A}$ itself is compatible with $\mathbf{S P}$, whereas the anonymity defined by all possible permutations on $\mathbb{N}$ comes in conflict with SP (van Liedekerke 1995; Lauwers 1997a). Furthermore, weakening QA to FA, it is possible to add SPC or SC as well. However, as shown in Proposition 1, if we strengthen the notion of impartiality FA to QA in such SWRs, we must go back to impossibility again. ${ }^{15}$ Therefore,

\footnotetext{
${ }^{14}$ See also Fleurbaey and Michel (2003) and Sakai (2008).

${ }^{15}$ Fleurbaey and Michel (2003) provide comprehensive analysis of the trade-offs between SP and some well-established anonymity axioms. They also obtain a similar impossibility to Proposition 1 with Limit Ranking. Limit Ranking is similar to our preference-continuity or consistency axioms but there is no logical relationship between them.
} 
under two basic principles, SP and FA, our choice of additional axioms QA or SPC (or SC) becomes a branching point in exploring admissible SWRs exhibiting higher level of comparability than the Suppes-Sen grading principle.

We now return to our main concern. We will formulate $\mathcal{Q}$-anonymous extensions of the W-leximin SWR and the overtaking criterion respectively below. For this purpose, we begin with the definitions of the W-leximin SWR and the overtaking criteron respectively. Let $\succsim_{L}^{n}$ denote the finite-horizon leximin ordering defined on $\mathbb{R}^{n}$ for each $n \in \mathbb{N}$ : for all $\boldsymbol{x}^{-n}, \boldsymbol{y}^{-n} \in \mathbb{R}^{n}, \boldsymbol{x}^{-n} \succsim_{L}^{n} \boldsymbol{y}^{-n}$ if and only if $\left(x_{(1)}^{-n}, \ldots, x_{(n)}^{-n}\right)=$ $\left(y_{(1)}^{-n}, \ldots, y_{(n)}^{-n}\right)$ or there exists an integer $m<n$ such that $\left(x_{(1)}^{-n}, \ldots, x_{(m)}^{-n}\right)=\left(y_{(1)}^{-n}, \ldots, y_{(m)}^{-n}\right)$ and $x_{(m+1)}^{-n}>y_{(m+1)}^{-n}$.

The W-leximin relation $\succsim_{L w}$ is defined as: for all $\boldsymbol{x}, \boldsymbol{y} \in X$,

$$
\left\{\begin{array}{l}
\boldsymbol{x} \succ_{L w} \boldsymbol{y} \text { iff there exists } \bar{n} \in \mathbb{N} \text { such that } \boldsymbol{x}^{-n} \succ_{L}^{n} \boldsymbol{y}^{-n} \text { for all } n \geq \bar{n} ; \\
\boldsymbol{x} \sim_{L w} \boldsymbol{y} \text { iff there exists } \bar{n} \in \mathbb{N} \text { such that } \boldsymbol{x}^{-n} \sim_{L}^{n} \boldsymbol{y}^{-n} \text { for all } n \geq \bar{n} .
\end{array}\right.
$$

Similarly, the overtaking criterion $\succsim o$ is defined as: for all $\boldsymbol{x}, \boldsymbol{y} \in X$,

$$
\left\{\begin{array}{l}
\boldsymbol{x} \succ_{O} \boldsymbol{y} \text { iff there exists } \bar{n} \in \mathbb{N} \text { such that } \sum_{i=1}^{n} x_{i}>\sum_{i=1}^{n} y_{i} \text { for all } n \geq \bar{n} ; \\
\boldsymbol{x} \sim_{O} \boldsymbol{y} \text { iff there exists } \bar{n} \in \mathbb{N} \text { such that } \sum_{i=1}^{n} x_{i}=\sum_{i=1}^{n} y_{i} \text { for all } n \geq \bar{n} .
\end{array}\right.
$$

We formally state the characterizations of $\succsim_{L w}$ and ${ }_{O}$ established by Asheim and Tungodden (2004) and Basu and Mitra (2007), which will be used to prove our main results later. ${ }^{16}$

Proposition 2 (Asheim and Tungodden 2004, Proposition 2). A SWR $\succsim$ satisfies $\boldsymbol{S P}$, $\boldsymbol{F A}$, any of $\{\boldsymbol{W P C}, \boldsymbol{W C}\}$, and $\boldsymbol{H E}$ if and only if $\succsim_{L w}$ is a subrelation of $\succsim$.

Proposition 3 (Asheim and Tungodden 2004, Proposition 5; Basu and Mitra 2007, Theorem 3). A SWR $\succsim$ satisfies SP, FA, any of $\{\boldsymbol{W P C}, \boldsymbol{W C}\}$, and any of $\{2 \boldsymbol{U C}, \boldsymbol{P U C}\}$ if and only if $\succsim O$ is a subrelation of $\succsim$.

We now introduce $\mathcal{Q}$-anonymous extensions of $\succsim_{L w}$ and $\succsim_{O}$, which we will call $\mathcal{Q}$-W-leximin $S W R$ and $\mathcal{Q}$-overtaking criterion respectively. The $\mathcal{Q}$-W-leximin relation, denoted by $\succsim_{Q L w}$, is defined as follows: for all $\boldsymbol{x}, \boldsymbol{y} \in X$,

$$
\boldsymbol{x} \succsim_{Q L w} \boldsymbol{y} \text { iff there exist } \boldsymbol{P}, \boldsymbol{Q} \in \mathcal{Q} \text { such that } \boldsymbol{P} \boldsymbol{x} \succsim_{L w} \boldsymbol{Q} \boldsymbol{y} \text {. }
$$

\footnotetext{
${ }^{16}$ In their original characterizations, Asheim and Tungodden (2004) use WPC and 2UC and Basu and Mitra (2007) employ WC and PUC. It is easily checked that WPC and WC are interchangeable and so are the two invariance axioms 2UC and PUC. In the statements of Propositions 2 and 3 and Theorems 1 and 2, we follow d'Aspremont and Gevers (2002) and use the expression "any of $\{\ldots\}$ " to mean the axioms in the braces are interchangeable.
} 
Similarly, the $\mathcal{Q}$-overtaking criterion, $\succsim_{Q O}$, is defined as: for all $\boldsymbol{x}, \boldsymbol{y} \in X$,

$$
\boldsymbol{x} \succsim_{Q O} \boldsymbol{y} \text { iff there exist } \boldsymbol{P}, \boldsymbol{Q} \in \mathcal{Q} \text { such that } \boldsymbol{P} \boldsymbol{x} \succsim_{O} \boldsymbol{Q} \boldsymbol{y} \text {. }
$$

The following proposition tells that each of $\succsim_{Q L w}$ and $\succsim_{Q O}$ is well-defined as a SWR on $X$ and the strict relation and the indifference relation corresponding to them are more simply characterized.

Proposition 4. Each of $\succsim_{Q L w}$ and $\succsim_{Q O}$ is well-defined as a SWR on X, i.e. reflexive and transitive, and satisfies the following: for all $\boldsymbol{x}, \boldsymbol{y} \in X$,

$$
\left\{\begin{array}{l}
\boldsymbol{x} \succ_{Q L w} \boldsymbol{y} \text { iff there exist } \boldsymbol{P}, \boldsymbol{Q} \in \mathcal{Q} \text { such that } \boldsymbol{P} \boldsymbol{x} \succ_{L w} \boldsymbol{Q y} \\
\boldsymbol{x} \sim_{Q L w} \boldsymbol{y} \text { iff there exists } \boldsymbol{P} \in \mathcal{Q} \text { such that } \boldsymbol{P} \boldsymbol{x} \sim_{L w} \boldsymbol{y}
\end{array}\right.
$$

and

$$
\left\{\begin{array}{l}
\boldsymbol{x} \succ_{Q O} \boldsymbol{y} \text { iff there exist } \boldsymbol{P}, \boldsymbol{Q} \in \mathcal{Q} \text { such that } \boldsymbol{P} \boldsymbol{x} \succ_{O} \boldsymbol{Q} \boldsymbol{y} \\
\boldsymbol{x} \sim_{Q O} \boldsymbol{y} \text { iff there exists } \boldsymbol{P} \in \mathcal{Q} \text { such that } \boldsymbol{P} \boldsymbol{x} \sim_{O} \boldsymbol{y}
\end{array}\right.
$$

Proof. See Appendix.

By (3b) and (4b), $\succsim_{Q L w}$ and $\succsim_{Q O}$ satisfy QA..${ }^{17}$ Furthermore, from (3a), (3b) and the fact that $\boldsymbol{I} \in \mathcal{Q}$, it follows that $\succsim_{L w}$ is a subrelation of $\succsim_{Q L w}$, and the same is true for $\succsim_{O}$ and $\succsim_{Q O}$ by (4a) and (4b). Thus, from Propositions 2 and $3, \succsim_{Q L w}$ and $\succsim_{Q O}$ also satisfy all the axioms characterizing $\succsim_{L w}$ and $\succsim_{O}$ respectively. Therefore, $\succsim_{Q L w}$ and $\succsim_{Q O}$ certainly belong to $\mathcal{Q}$-anonymous subclasses of those characterized in Propositions 2 and 3.

Our main results show that the classes of all SWRs satisfying both QA and WPC (and WC) as well as the basic axioms (i.e. the shaded area in Figure 1) coincide with all SWRs that include $\succsim_{Q L w}$ and $\succsim_{Q O}$ respectively as a subrelation.

Theorem 1. A SWR $\succsim$ satisfies $\boldsymbol{S P}, \boldsymbol{Q A}$, any of $\{\boldsymbol{W P C}, \boldsymbol{W C}\}$, and $\boldsymbol{H E}$ if and only if $\succsim_{Q L w}$ is a subrelation of $\succsim$.

Proof. See Appendix.

Theorem 2. A SWR $\succsim$ satisfies SP, $Q A$, any of $\{\boldsymbol{W P C}, \boldsymbol{W C}\}$, and any of $\{2 \boldsymbol{2 U C}, \boldsymbol{P U C}\}$ if and only if $\succsim_{Q O}$ is a subrelation of $\succsim$.

Proof. See Appendix.

\footnotetext{
${ }^{17}$ Notice that $\boldsymbol{P} \boldsymbol{x} \sim_{Q L w} \boldsymbol{x}$ follows from the fact that $(\boldsymbol{x}=) \boldsymbol{P}^{\prime} \boldsymbol{P} \boldsymbol{x} \sim_{L w} \boldsymbol{x}$.
} 
Table 1: Characterizations of $\mathcal{F}$-anonymous SWRs and $\mathcal{Q}$-extensions

\begin{tabular}{l|ccccccc|l}
\hline \hline \multirow{2}{*}{$\begin{array}{l}\text { SWR } \\
\text { (least restrictive) }\end{array}$} & SP & FA & QA & SPC/SC & WPC/WC & HE & 2UC/PUC & \\
\hline C-W-leximin & $\oplus$ & + & $\oplus$ & - & $\oplus$ & $\oplus$ & - & Theoracterization \\
W-leximin & $\oplus$ & $\oplus$ & & & $\oplus$ & $\oplus$ & - & AT (2004) \\
S-leximin & $\oplus$ & $\oplus$ & - & $\oplus$ & + & $\oplus$ & - & AT (2004) \\
\hline $\mathcal{Q}$-overtaking & $\oplus$ & + & $\oplus$ & - & $\oplus$ & - & $\oplus$ & Theorem 2 \\
Overtaking & $\oplus$ & $\oplus$ & & & $\oplus$ & - & $\oplus$ & AT (2004) and BM (2007) \\
Catching-up & $\oplus$ & $\oplus$ & - & $\oplus$ & + & - & $\oplus$ & AT (2004) and BM (2007) \\
\hline \hline
\end{tabular}

Theorem 1 (resp. 2) is interpreted as saying that $\succsim_{Q L w}$ (resp. $\succsim_{Q O}$ ) is the least restrictive SWR among all SWRs satisfying SP, QA, WPC (or WC), and HE (resp. 2UC (or PUC)). Formally, for all $\boldsymbol{x}, \boldsymbol{y} \in X$, we have

$$
\left\{\begin{array}{l}
\boldsymbol{x} \succsim_{Q L w} \boldsymbol{y} \text { if and only if } \boldsymbol{x} \succsim \boldsymbol{y} \text { for all } \succsim \in \Xi_{Q L w} \\
\boldsymbol{x} \succsim_{Q O} \boldsymbol{y} \text { if and only if } \boldsymbol{x} \succsim \boldsymbol{y} \text { for all } \succsim \in \Xi_{Q O}
\end{array}\right.
$$

where $\Xi_{Q L w}$ (resp. $\Xi_{Q O}$ ) is the set of all SWRs that satisfy SP, QA, any of $\{$ WPC, WC $\}$, and HE (resp. any of $\{$ 2UC, PUC $\}$ ). ${ }^{18}$ From Arrow's (1963) variant of Szpilrajn's (1930) lemma, each of $\Xi_{Q L w}$ and $\Xi_{Q O}$ contains at least one complete SWR, i.e. social welfare ordering. ${ }^{19}$

Table 1 summarizes the characterizations in Theorems 1 and 2 and compares them with those established by Asheim and Tungodden (2004) and Basu and Mitra (2007) (in Table 1, AT (2004) and BM (2007) respectively). For each row in Table 1, the class of SWRs that includes the SWR stated in the first column as a subrelation is characterized by the axioms indicated by $\oplus$, and furthermore, each SWR out of the class satisfies (resp. violates) the axioms indicated by + (resp. - ). Compared to the characterizations in Asheim and Tungodden (2004) and Basu and Mitra (2007), our results are regarded as the refinements of admissible SWRs by using the stronger notion of impartiality, QA, than FA. The impossibilities in Proposition 1 give "_" in the 4th and 5th columns in Table 1. Consequently, it can be said that it is possible to additionally impose the stronger notion of impartiality $\mathbf{Q A}$, but it comes at the cost of the stronger versions of Preference-continuity and Consistency, SPC and SC.

\footnotetext{
${ }^{18}$ For each of the two equivalence assertions, the only if part follows from the only if statement of the corresponding theorem, and the if part is also straightforward from the fact that $\succsim_{Q L w} \in \Xi_{Q L w}$ (resp. $\left.\succsim_{Q O} \in \Xi_{Q O}\right)$.

${ }^{19}$ However, these orderings cannot have an explicit description (Lauwers 2006; Zame 2007).
} 


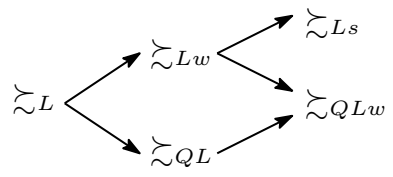

Figure 2: Extended leximin SWRs

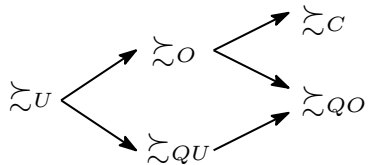

Figure 3: Extended utilitarian SWRs

\section{Comparison with some well-established SWRs}

In this section, we compare our new SWRs $\succsim_{Q L w}$ and $\succsim_{Q O}$ with some relevant ones in the literature. We begin with the formal definitions of the utilitarian SWR (Basu and Mitra 2007) and the leximin SWR (Bossert et al. 2007) and also of their $\mathcal{Q}$-anonymous extensions, $\mathcal{Q}$-utilitarian SWR (Banerjee 2006) and $\mathcal{Q}$-leximin SWR (Kamaga and Kojima 2008).

- The $\mathcal{Q}$-leximin SWR ${ }_{Q L}$ and the $\mathcal{Q}$-utilitarian SWR ${ }_{Q U}$ :

$\boldsymbol{x} \succsim_{Q L} \boldsymbol{y}$ iff there exists $\boldsymbol{P} \in \mathcal{Q}$ such that $\boldsymbol{P} \boldsymbol{x} \succsim_{L} \boldsymbol{y}$.

$\boldsymbol{x} \succsim_{Q U} \boldsymbol{y}$ iff there exists $\boldsymbol{P} \in \mathcal{Q}$ such that $\boldsymbol{P} \boldsymbol{x} \succsim_{U} \boldsymbol{y}$.

- The leximin SWR $\succsim_{L}$ and the utilitarian SWR $\succsim_{U}$ :

$\boldsymbol{x} \succsim_{L} \boldsymbol{y}$ iff there exists $n \in \mathbb{N}$ such that $\boldsymbol{x}^{-n} \succsim_{L}^{n} \boldsymbol{y}^{-n}$ and $\boldsymbol{x}^{+n} \geqslant \boldsymbol{y}^{+n}$.

$\boldsymbol{x} \succsim_{U} \boldsymbol{y}$ iff there exists $n \in \mathbb{N}$ such that $\sum_{i=1}^{n} x_{i} \geq \sum_{i=1}^{n} y_{i}$ and $\boldsymbol{x}^{+n} \geqslant \boldsymbol{y}^{+n}$.

Figures 2 and 3 summarize the relationships among the SWRs we discussed so far, where $\succsim_{L s}$ and $\succsim_{C}$ denote the S-leximin SWR and the catching-up criterion respectively and we write $\succsim_{A} \rightarrow_{{ }_{B}}$ to mean $\succsim_{A}$ is a subrelation of $\succsim_{B} \cdot{ }^{20}$

The following example shows that our new SWRs $\succsim_{Q L w}$ and $\succsim_{Q O}$ respectively can make further comparisons of streams beyond the limits of their subrelations $\succsim_{L}$, $\succsim_{U}, \succsim_{L w}, \succsim_{Q L}, \succsim_{O}$, and $\succsim_{Q U}$.

Example 1. Consider the following utility streams $\boldsymbol{x}$ and $\boldsymbol{y}$ :

$$
\begin{aligned}
& \boldsymbol{x}=\left(1,1, \frac{1}{3}, \frac{1}{3}, \frac{1}{3^{2}}, \frac{1}{3^{2}}, \frac{1}{3^{3}}, \ldots\right) \\
& \boldsymbol{y}=\left(1, \frac{2}{3}, \frac{2}{3}, \frac{2}{3^{2}}, \frac{2}{3^{2}}, \frac{2}{3^{3}}, \frac{2}{3^{3}}, \ldots\right) .
\end{aligned}
$$

\footnotetext{
${ }^{20}$ The S-leximin SWR $\succsim_{L s}$ and the catching-up criterion $\succsim_{C}$ are defined as: $\boldsymbol{x} \succsim_{L s} \boldsymbol{y}$ iff there exists $\bar{n} \in \mathbb{N}$ such that $\boldsymbol{x}^{-n} \succsim_{L}^{n} \boldsymbol{y}^{-n}$ for all $n \geq \bar{n} ; \boldsymbol{x} \succsim_{C} \boldsymbol{y}$ iff there exists $\bar{n} \in \mathbb{N}$ such that $\sum_{i=1}^{n} x_{i} \geq$ $\sum_{i=1}^{n} y_{i}$ for all $n \geq \bar{n}$.
} 
One can generate the streams $\boldsymbol{x}$ and $\boldsymbol{y}$ in the following way:

$$
\left(x_{n}, y_{n}\right)= \begin{cases}(1,1) & \text { if } n=1 \\ \left(\frac{3}{\sqrt{3}^{n}}, \frac{2}{\sqrt{3}^{n}}\right) & \text { if } n \text { is even } \\ \left(\frac{\sqrt{3}}{\sqrt{3}^{n}}, \frac{2 \sqrt{3}^{3}}{\sqrt{3}^{n}}\right) & \text { otherwise. }\end{cases}
$$

Clearly, $x$ and $y$ are non-comparable according to $\succsim_{L w}$, since

$$
\begin{cases}\min \left\{x_{1}, \ldots, x_{n}\right\}<\min \left\{y_{1}, \ldots, y_{n}\right\} & \text { for all even } n \\ \min \left\{x_{1}, \ldots, x_{n}\right\}>\min \left\{y_{1}, \ldots, y_{n}\right\} & \text { for all odd } n>1\end{cases}
$$

Moreover, $\succsim o$ also declares them non-comparable, since

$$
\begin{cases}\sum_{i=1}^{n} x_{i}>\sum_{i=1}^{n} y_{i} & \text { for all even } n, \\ \sum_{i=1}^{n} x_{i}=\sum_{i=1}^{n} y_{i} & \text { for all odd } n .\end{cases}
$$

Since any permutation $\boldsymbol{P}$ in $\mathcal{Q}$ cannot give the Pareto dominance between $\boldsymbol{P} \boldsymbol{x}$ and $\boldsymbol{y}, \succsim_{Q L}$ and $\succsim_{Q U}$ still declare $\boldsymbol{x}$ and $\boldsymbol{y}$ non-comparable. Thus, $\boldsymbol{x}$ and $\boldsymbol{y}$ are noncomparable according to any of $\succsim_{L w}, \succsim_{Q L}, \succsim_{O}$, and $\succsim_{Q U}$ (thus, $\succsim_{L}$ and $\succsim_{U}$ either).

However, using the 2-period cyclic permutation $\overline{\boldsymbol{P}} \in \mathcal{Q}$ corresponding to the permutation $\pi$ defined as: $\pi(n)=n+1$ if $n$ is odd, and $\pi(n)=n-1$ if $n$ is even, we have $\boldsymbol{x} \succ_{L w} \overline{\boldsymbol{P}} \boldsymbol{y}$ and $\boldsymbol{x} \succ_{O} \overline{\boldsymbol{P}} \boldsymbol{y}$. Thus, according to $\succsim_{Q L w}$ or $\succsim_{Q O}, \boldsymbol{x}$ and $\boldsymbol{y}$ are comparable and $\boldsymbol{x} \succ_{Q L w} \boldsymbol{y}$ and $\boldsymbol{x} \succ_{Q O} \boldsymbol{y}$.

The streams $\boldsymbol{x}$ and $\overline{\boldsymbol{P}} \boldsymbol{y}$ can be an example of the case where $\succsim_{L w}$ and $\succsim o$ can compare those streams, but $\succsim_{Q L}$ and $\succsim_{Q U}$ cannot. As noted in the introduction, the streams $(1,0,1,0, \ldots)$ and $(0,1,0,1, \ldots)$ give us an example of the converse case. Since our

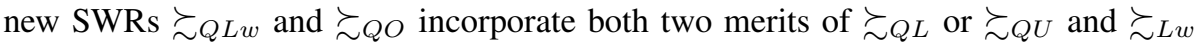
or $\succsim O$ respectively, they can resolve the trade-off in the choice of the $\mathcal{Q}$-anonymous extensions $\succsim_{Q L}$ and $\succsim_{Q U}$ or the preference-continuous or consistent relations $\succsim_{L w}$ and $\succsim o$.

\section{Concluding remarks}

We have characterized the classes of all SWRs satisfying not only the basic axioms which give axiomatic foundations of the infinite-horizon variants of the leximin principle and utilitarianism, $\succsim_{L}$ and $\succsim_{U}$, respectively (SP, FA and HE or $2 \mathbf{U C}$ (or PUC)) but also the two additional requirements, the weak version of preference-continuity or consistency (WPC or WC) and the stronger notion of impartiality than Finite Anonymity 
(QA). In these classes of SWRs, our new extended SWRs $\succsim_{Q L w}$ and $\succsim_{Q O}$ respectively are the least restrictive ones. Therefore, our two characterization theorems tell that under the axioms stated above, our evaluation of intergenerational welfare distributions must be based on the comparisons according to $\succsim_{Q L w}$ and $\succsim_{Q O}$ respectively. As we have observed in Sect. $4, \succsim_{Q L w}$ and $\succsim_{Q O}$ can lead us to further comparisons of streams beyond the limits of the well-established extended SWRs $\succsim_{L w}, \succsim_{O}, \succsim_{Q L}$ and $\succsim_{Q U}$.

Both $\succsim_{Q L w}$ and $\succsim_{Q O}$ are formulated as the extensions of $\succsim_{L w}$ and $\succsim_{O}$ by using permutations of the class $\mathcal{Q}$ and are characterized by strengthening $\mathbf{F A}$ to $\mathbf{Q A}$ in the lists of the axioms characterizing $\succsim_{L w}$ and $\succsim_{O}$ respectively. As will be shown in Appendix B, these results can be generalized to any SWR defined by using a sequence of finite-horizon orderings satisfying certain moderate properties in the same way as in $\succsim_{L w}$ and $\succsim_{O}$. Such a general approach to the analysis of infinite-horizon criteria is initiated by d'Aspremont (2007) and is also taken by Asheim et al. (2008), Kamaga and Kojima (2008) and Sakai (2008).

Finally, we should discuss the issue, raised by Banerjee (2006), on the rankings of summable streams derived by extended utilitarian SWRs. As discussed in Example 3 in Banerjee (2006), $\succsim_{Q U}$ declares the following two summable sequences $\boldsymbol{u}$ and $\boldsymbol{v}$ to be non-comparable: $\boldsymbol{u}=\left(1, \frac{1}{2}, \frac{1}{2}, \frac{1}{2^{3}}, \frac{1}{2^{3}}, \ldots\right)$ and $\boldsymbol{v}=\left(1,1, \frac{1}{2^{2}}, \frac{1}{2^{2}}, \frac{1}{2^{4}}, \ldots\right)$. Since we have $\sum_{i=1}^{\infty} u_{i}=7 / 3<8 / 3=\sum_{i=1}^{\infty} v_{i}$, if we follow the spirit of utilitarianism, then we should conclude $\boldsymbol{v}$ is strictly better than $\boldsymbol{u}$. Our extended utilitarian relation $\succsim_{Q O}$, which satisfies WPC (and WC), can compare any two summable sequences in terms of their sums of utilities if their utility sums are different. ${ }^{21}$ In this respect, $\succsim_{Q O}$ is a quite appealing infinite-horizon formulation of utilitarianism. However, it still fails to rank summable sequences according to their sums of utilities if the total sums are equal. Notice that $\boldsymbol{x}$ and $\boldsymbol{y}$ considered in Sect. 4 are summable and $\sum_{i=1}^{\infty} x_{i}=$ $\sum_{i=1}^{\infty} y_{i}=3$. For these streams, $\succsim_{Q O}$ concludes that $\boldsymbol{x}$ is strictly better than $\boldsymbol{y}$. To formulate and characterize an extended utilitarian SWR that completely reflects the utilitarian doctrine for all summable sequences, we must lay down WPC and WC. We leave this issue for future research.

\footnotetext{
${ }^{21}$ This result can be generalized to any two sequences such that the cumulative sums of difference between the streams converge in $\mathbb{R}_{++}$.
} 


\section{Appendix}

\section{A: Proof of Proposition 1}

Proof of Proposition 1. First, we prove (i) by contradiction. Suppose that $\succsim$ satisfies SP, QA, and SPC. Let $\boldsymbol{x}=(1,0,1,0, \ldots)$ and $\boldsymbol{y}=(0,1,0,1, \ldots)$. By QA,

$$
\left(\boldsymbol{x}^{-n}, \boldsymbol{y}^{+n}\right) \sim \boldsymbol{y} \text { for all even } n \in \mathbb{N},
$$

and also $\left(\boldsymbol{x}^{-n}, \boldsymbol{y}^{+n}\right) \sim\left(x_{1}, \boldsymbol{y}^{+1}\right)$ for all odd $n \in \mathbb{N}$. By $\mathbf{S P},\left(x_{1}, \boldsymbol{y}^{+1}\right) \succ \boldsymbol{y}$. By transitivity,

$$
\left(\boldsymbol{x}^{-n}, \boldsymbol{y}^{+n}\right) \succ \boldsymbol{y} \text { for all odd } n \in \mathbb{N} \text {. }
$$

From (5) and (6), SPC gives $\boldsymbol{x} \succ \boldsymbol{y}$, while $\boldsymbol{x} \sim \boldsymbol{y}$ is obtained by QA.

Next, we prove (ii). The proof is similar to that of (i). Suppose $\succsim$ satisfies SP, QA, SC. Let $\boldsymbol{x}=(1,0,1,0, \ldots)$ and $\boldsymbol{y}=(0,1,0,1, \ldots)$. By $\mathbf{Q A}$,

$$
\left(\boldsymbol{x}^{-n}, 0,0, \ldots\right) \sim\left(\boldsymbol{y}^{-n}, 0,0, \ldots\right) \text { for all even } n \in \mathbb{N}
$$

and $\left(\boldsymbol{x}^{-n}, 0,0, \ldots\right) \sim\left(\boldsymbol{y}^{-(n+2)}, 0,0, \ldots\right)$ for all odd $n \in \mathbb{N}$. By $\mathbf{S P},\left(\boldsymbol{x}^{-(n+2)}, 0,0, \ldots\right) \succ$ $\left(\boldsymbol{x}^{-n}, 0,0, \ldots\right)$ for all $n \in \mathbb{N}$. Since $\succsim$ is transitive,

$$
\left(\boldsymbol{x}^{-n}, 0,0, \ldots\right) \succ\left(\boldsymbol{y}^{-n}, 0,0, \ldots\right) \text { for all odd } n \geq 3
$$

From (7) and (8), SC gives $\boldsymbol{x} \succ \boldsymbol{y}$, while, by QA, $\boldsymbol{x} \sim \boldsymbol{y}$.

\section{B: Proof of Proposition 4}

Let $\succsim_{U}^{n}$ denote the finite-horizon utilitarian relation defined on $\mathbb{R}^{n}$ for each $n \in \mathbb{N}$ : for all $x^{-n}, y^{-n} \in \mathbb{R}^{n}, x^{-n} \succsim_{U}^{n} y^{-n}$ if and only if $\sum_{i=1}^{n} x_{i} \geq \sum_{i=1}^{n} y_{i}$. Note that both of the finite-horizon leximin and utilitarian relations $\succsim_{L}^{n}$ and $\succsim_{U}^{n}$ are orderings on $\mathbb{R}^{n}$ for all $n \in \mathbb{N}$, and moreover, each of the sequences of them, $\left\{\succsim_{L}^{n}\right\}_{n \in \mathbb{N}}$ and $\left\{\succsim_{U}^{n}\right\}_{n \in \mathbb{N}}$, satisfies the following three properties: ${ }^{22}$ for all $n \in \mathbb{N}$ and all $\boldsymbol{x}^{-n}, \boldsymbol{y}^{-n} \in \mathbb{R}^{n}$,

P1: If $\boldsymbol{x}^{-n}>\boldsymbol{y}^{-n}$, then $\boldsymbol{x}^{-n} \succ^{n} \boldsymbol{y}^{-n}$;

P2: If $\boldsymbol{x}^{-n}$ is a permutation of $\boldsymbol{y}^{-n}$, then $\boldsymbol{x}^{-n} \sim^{n} \boldsymbol{y}^{-n}$;

P3: For all $r \in \mathbb{R},\left(\boldsymbol{x}^{-n}, r\right) \succsim^{n+1}\left(\boldsymbol{y}^{-n}, r\right)$ if and only if $\boldsymbol{x}^{-n} \succsim^{n} \boldsymbol{y}^{-n}$,

${ }^{22} \mathbf{P 1}$ is the finite-horizon version of SP. P2 is a well-known anonymity axiom in a finite-horizon framework. $\mathbf{P 3}$ is a kind of separability requirement similar to Extended Independence of the Utilities of Unconcerned Individuals introduced by Blackorby et al. (2002) in the framework of variable population social choice, which requires our evaluation to be independent of the existence of an unconcerned generation. 
where $\succsim^{n}$ denotes an ordering on $\mathbb{R}^{n}$ for all $n \in \mathbb{N}$.

We provide the proof of Proposition 4 for the case of $\succsim Q L w$ by only using the properties $\mathbf{P 1}, \mathbf{P 2}$, and $\mathbf{P 3}$. Thus, the same argument can be directly applied to the case of $\succsim_{Q O}$, and we omit it.

First, we prove the equivalence assertions in (3a) and (3b). To prove them, we use the following lemma.

Lemma 1. For all $\boldsymbol{x}, \boldsymbol{y} \in X$ and all $\boldsymbol{P} \in \mathcal{Q}$,

$$
\boldsymbol{x} \sim_{L w} \boldsymbol{y} \text { if and only if } \boldsymbol{P} \boldsymbol{x} \sim_{L w} \boldsymbol{P} \boldsymbol{y} .
$$

Proof. (only if part) Assume $\boldsymbol{x} \sim_{L w} \boldsymbol{y}$, and consider any $\boldsymbol{P} \in \mathcal{Q}$. Since $\boldsymbol{P} \in \mathcal{Q}$, there exists $k \in \mathbb{N}$ such that for all $n \in \mathbb{N}, \boldsymbol{P}(n k)$ is a finite-dimensional permutation matrix. By the definition of $\succsim_{L w}$, we can find $\bar{m} \in \mathbb{N}$ such that

$$
\boldsymbol{x}^{-m} \sim_{L}^{m} \boldsymbol{y}^{-m} \text { for all } m \geq \bar{m} \text { with } \bar{m}=n k \text { for some } n \in \mathbb{N} \text {. }
$$

We show, by contradiction, that

$$
x_{m}=y_{m} \text { for all } m>\bar{m} .
$$

Suppose that (11) does not hold. Let $m^{\prime}$ be the smallest integer such that $m^{\prime}>$ $\bar{m}$ and $x_{m^{\prime}} \neq y_{m^{\prime}}$. Without loss of generality, we assume $x_{m^{\prime}}>y_{m^{\prime}}$. By P3, $\left(\boldsymbol{x}^{-\left(m^{\prime}-1\right)}, y_{m^{\prime}}\right) \sim_{L}^{m^{\prime}} \boldsymbol{y}^{-m^{\prime}}$. By P1, $\boldsymbol{x}^{-m^{\prime}} \succ_{L}^{m^{\prime}}\left(\boldsymbol{x}^{-\left(m^{\prime}-1\right)}, y_{m^{\prime}}\right)$. The transitivity of $\succsim_{L}^{m^{\prime}}$ gives $\boldsymbol{x}^{-m^{\prime}} \succ_{L}^{m^{\prime}} \boldsymbol{y}^{-m^{\prime}}$, which contradicts (10). Thus, (11) holds. Since $\boldsymbol{P}(\bar{m})$ is a finite-dimensional permutation matrix, by $\mathbf{P} 2, \boldsymbol{x}^{-\bar{m}} \sim_{L}^{\bar{m}}(\boldsymbol{P} \boldsymbol{x})^{-\bar{m}}$ and $\boldsymbol{y}^{-\bar{m}} \sim_{L}^{\bar{m}}(\boldsymbol{P} \boldsymbol{y})^{-\bar{m}}$. Then, by the transitivity of $\succsim^{\bar{m}}$ and $(10),(\boldsymbol{P} \boldsymbol{x})^{-\bar{m}} \sim_{L}^{\bar{m}}(\boldsymbol{P} \boldsymbol{y})^{-\bar{m}}$. Note that, by (11), $(\boldsymbol{P} \boldsymbol{x})^{+\bar{m}}=(\boldsymbol{P} \boldsymbol{y})^{+\bar{m}}$. Thus, by $\mathbf{P 3},(\boldsymbol{P} \boldsymbol{x})^{-m} \sim_{L}^{m}(\boldsymbol{P} \boldsymbol{y})^{-m}$ holds for all $m \geq \bar{m}$. By the definition of $\succsim_{L w}, \boldsymbol{P} \boldsymbol{x} \sim_{L w} \boldsymbol{P} \boldsymbol{y}$.

(if part) Take any $\boldsymbol{x}, \boldsymbol{y} \in X$ and any $\boldsymbol{P} \in \mathcal{Q}$, and assume $\boldsymbol{P} \boldsymbol{x} \sim_{L w} \boldsymbol{P} \boldsymbol{y}$. Since $\boldsymbol{P}^{\prime} \in \mathcal{Q}$, the only if part of the lemma gives $(\boldsymbol{x}=) \boldsymbol{P}^{\prime} \boldsymbol{P} \boldsymbol{x} \sim_{L w} \boldsymbol{P}^{\prime} \boldsymbol{P} \boldsymbol{y}(=\boldsymbol{y})$.

We are ready to prove the equivalence assertions in (3a) and (3b).

(only if (3a)): Suppose $\boldsymbol{x} \succ_{Q L w} \boldsymbol{y}$. Then, by definition, there exist $\boldsymbol{P}, \boldsymbol{Q} \in \mathcal{Q}$ such that $\boldsymbol{P} \boldsymbol{x} \succsim_{L w} \boldsymbol{Q} \boldsymbol{y}$. Moreover, for all $\boldsymbol{R}, \boldsymbol{S} \in \mathcal{Q}, \neg\left(\boldsymbol{R} \boldsymbol{y} \succsim_{L w} \boldsymbol{S} \boldsymbol{x}\right)$. Otherwise, we have a contradiction to $\boldsymbol{x} \succ_{Q L w} \boldsymbol{y}$. Thus, $\neg\left(\boldsymbol{Q} \boldsymbol{y} \succsim_{L w} \boldsymbol{P} \boldsymbol{x}\right)$.

(if (3a)): Suppose that there exist $\boldsymbol{P}, \boldsymbol{Q} \in \mathcal{Q}$ such that $\boldsymbol{P} \boldsymbol{x} \succ_{L w} \boldsymbol{Q y}$. Then, by definition, $\boldsymbol{x} \succsim_{Q L w} \boldsymbol{y}$. We show, by contradiction, that $\neg\left(\boldsymbol{y} \succsim_{Q L w} \boldsymbol{x}\right)$. Assume $\boldsymbol{y} \succsim_{Q L w} \boldsymbol{x}$. Then, there exist $\boldsymbol{R}, \boldsymbol{S} \in \mathcal{Q}$ such that $\boldsymbol{R} \boldsymbol{y} \succsim_{L w} \boldsymbol{S} \boldsymbol{x}$. Let $p, q, r, s \in \mathbb{N}$ be period of cycle in $\boldsymbol{P}, \boldsymbol{Q}, \boldsymbol{R}$, and $\boldsymbol{S}$ respectively. By the definition of $\succsim_{L w}$, there exist 
$\bar{n}, \bar{n}^{\prime} \in \mathbb{N}$ such that

$$
\boldsymbol{P} \boldsymbol{x}^{-n} \succ_{L}^{n} \boldsymbol{Q} \boldsymbol{y}^{-n} \text { for all } n \geq \bar{n}
$$

and

$$
\left\{\begin{array}{l}
\boldsymbol{R} \boldsymbol{y}^{-n} \succ_{L}^{n} \boldsymbol{S} \boldsymbol{x}^{-n} \text { for all } n \geq \bar{n}^{\prime} \\
\text { or } \\
\boldsymbol{R} \boldsymbol{y}^{-n} \sim_{L}^{n} \boldsymbol{S} \boldsymbol{x}^{-n} \text { for all } n \geq \bar{n}^{\prime}
\end{array}\right.
$$

Let $k=p \times q \times r \times s$, and choose $\hat{n} \in \mathbb{N}$ such that $\hat{n} k \geq \max \left\{\bar{n}, \bar{n}^{\prime}\right\}$. Note that $\boldsymbol{P}(\hat{n} k), \boldsymbol{Q}(\hat{n} k), \boldsymbol{R}(\hat{n} k)$, and $\boldsymbol{S}(\hat{n} k)$ are finite-dimensional permutation matrices. By $\mathbf{P 2}, \boldsymbol{Q} \boldsymbol{y}^{-\hat{n} k} \sim_{L}^{\hat{n} k} \boldsymbol{R} \boldsymbol{y}^{-\hat{n} k}$. Then, from (12) and (13), the transitivity of $\succsim^{\hat{n} k}$ gives $\boldsymbol{P} \boldsymbol{x}^{-\hat{n} k} \succ_{L}^{\hat{n} k} \boldsymbol{S} \boldsymbol{x}^{-\hat{n} k}$, which contradicts $\mathbf{P 2}$.

(only if (3b)): Suppose $\boldsymbol{x} \sim_{Q L w} \boldsymbol{y}$. By definition, there exist $\boldsymbol{P}, \boldsymbol{Q} \in \mathcal{Q}$ such that $\boldsymbol{P} \boldsymbol{x} \succsim_{L w} \boldsymbol{Q} \boldsymbol{y}$. If we have $\neg\left(\boldsymbol{Q} \boldsymbol{y} \succsim_{L w} \boldsymbol{P} \boldsymbol{x}\right)$, then, by (3a), $\boldsymbol{x} \succ_{Q L w} \boldsymbol{y}$, and a contradiction is obtained. Thus, $\boldsymbol{Q} \boldsymbol{y} \succsim_{L w} \boldsymbol{P} \boldsymbol{x}$ must hold, and $\boldsymbol{P} \boldsymbol{x} \sim_{L w} \boldsymbol{Q} \boldsymbol{y}$ follows. By Lemma 1, $\boldsymbol{Q}^{\prime} \boldsymbol{P} \boldsymbol{x} \sim_{L w} \boldsymbol{Q}^{\prime} \boldsymbol{Q} \boldsymbol{y}(=\boldsymbol{y})$. Since $\boldsymbol{Q}^{\prime} \boldsymbol{P} \in \mathcal{Q}$, the proof is completed.

(if (3b)): Suppose that there exists $\boldsymbol{P} \in \mathcal{Q}$ such that $\boldsymbol{P} \boldsymbol{x} \sim_{L w} \boldsymbol{y}$. Since $\boldsymbol{P}, \boldsymbol{I} \in$ $\mathcal{Q}, \boldsymbol{x} \succsim_{Q L w} \boldsymbol{y}$. If we have $\neg\left(\boldsymbol{y} \succsim_{Q L w} \boldsymbol{x}\right)$, then, by definition, for all $\boldsymbol{Q}, \boldsymbol{R} \in \mathcal{Q}$, $\neg\left(\boldsymbol{Q} \boldsymbol{y} \succsim_{L w} \boldsymbol{R} \boldsymbol{x}\right)$, which contradicts $\boldsymbol{P} \boldsymbol{x} \sim_{L w} \boldsymbol{y}$. Thus, $\boldsymbol{y} \succsim_{Q L w} \boldsymbol{x}$.

Next, we prove that $\succsim_{Q L w}$ is a SWR on $X$. To prove this, we begin with the following lemma.

Lemma 2. $\succsim_{Q L w}$ is quasi-transitive, i.e. for all $\boldsymbol{x}, \boldsymbol{y}, \boldsymbol{z} \in X$, if $\boldsymbol{x} \succ_{Q L w} \boldsymbol{y}$ and $\boldsymbol{y} \succ_{Q L w} \boldsymbol{z}$, then $\boldsymbol{x} \succ_{Q L w} \boldsymbol{z}$.

Proof. Assume that $\boldsymbol{x} \succ_{Q L w} \boldsymbol{y}$ and $\boldsymbol{y} \succ_{Q L w} \boldsymbol{z}$. By (3a), there exist $\boldsymbol{P}, \boldsymbol{Q}, \boldsymbol{R}, \boldsymbol{S} \in \mathcal{Q}$ such that $\boldsymbol{P} \boldsymbol{x} \succ_{L w} \boldsymbol{Q} \boldsymbol{y}$ and $\boldsymbol{R} \boldsymbol{y} \succ_{L w} \boldsymbol{S} \boldsymbol{z}$. Let $p, q, r, s \in \mathbb{N}$ be period of cycle in $\boldsymbol{P}, \boldsymbol{Q}, \boldsymbol{R}$, and $\boldsymbol{S}$ respectively and also $k=p \times q \times r \times s$. Then, for all $n \in \mathbb{N}$, each of $\boldsymbol{P}(n k), \boldsymbol{Q}(n k), \boldsymbol{R}(n k)$, and $\boldsymbol{S}(n k)$ is a finite-dimensional permutation matrix. By the definition of $\succsim_{L w}$, we can find $\bar{m} \in \mathbb{N}$ such that $\bar{m}=n k$ for some $n \in \mathbb{N}$ and, for all $m \geq \bar{m}$,

$$
(\boldsymbol{P} \boldsymbol{x})^{-m} \succ_{L}^{m}(\boldsymbol{Q} \boldsymbol{y})^{-m} \text { and }(\boldsymbol{R} \boldsymbol{y})^{-m} \succ_{L}^{m}(\boldsymbol{S} \boldsymbol{z})^{-m}
$$

By P2, $(\boldsymbol{Q y})^{-N \bar{m}} \sim_{L}^{N \bar{m}}(\boldsymbol{R} \boldsymbol{y})^{-N \bar{m}}$ for all $N \in \mathbb{N}$. Then, by (14), the transitivity of 
$\succsim_{L}^{N \bar{m}}$ gives

$$
(\boldsymbol{P} \boldsymbol{x})^{-N \bar{m}} \succ_{L}^{N \bar{m}}(\boldsymbol{S} \boldsymbol{z})^{-N \bar{m}} \text { for all } N \in \mathbb{N} \text {. }
$$

We show that there exist $\tilde{\boldsymbol{P}}, \tilde{\boldsymbol{S}} \in \mathcal{Q}$ such that $(\tilde{\boldsymbol{P}} \boldsymbol{x})^{-m} \succ_{L}^{m}(\tilde{\boldsymbol{S}} \boldsymbol{z})^{-m}$ for all $m \geq$ $\bar{m}$. Then, by the definition of $\succsim_{L w}$ and (3a), $\boldsymbol{x} \succ_{Q L w} \boldsymbol{z}$ is obtained as desired. If $(\boldsymbol{P} \boldsymbol{x})^{-m} \succ_{L}^{m}(\boldsymbol{S} \boldsymbol{z})^{-m}$ for all $m \geq \bar{m}, \boldsymbol{P}=\tilde{\boldsymbol{P}}$ and $\boldsymbol{S}=\tilde{\boldsymbol{S}}$ trivially follow. We now consider the other cases. For any $N \in \mathbb{N}$, let $i(N) \in\{N \bar{m}+1, \ldots,(N+1) \bar{m}-1\}$ be the smallest integer for which $\left.\neg\left((\boldsymbol{P} \boldsymbol{x})^{-i(N)} \succ_{L}^{i(N)}(\boldsymbol{S} \boldsymbol{z})^{-i(N)}\right)\right)$ holds. Since $\succsim_{L}^{i(N)}$ is complete, this is equivalent to

$$
(\boldsymbol{S} \boldsymbol{z})^{-i(N)} \succsim_{L}^{i(N)}(\boldsymbol{P} \boldsymbol{x})^{-i(N)}
$$

By (15), there must be $j(N) \in\{i(N)+1, \ldots,(N+1) \bar{m}\}$ such that

$$
(P x)_{j(N)}>(S z)_{j(N)}
$$

Otherwise, by (16), $\mathbf{P 1}$ and P3, we have $(\boldsymbol{S} \boldsymbol{z})^{-(N+1) \bar{m}} \succsim_{L}^{(N+1) \bar{m}}(\boldsymbol{P} \boldsymbol{x})^{-(N+1) \bar{m}}$, which contradicts (15). We construct $\tilde{\boldsymbol{P}}$ and $\tilde{\boldsymbol{Q}}$ as follows. Let $\boldsymbol{T}_{1(N)} \in \mathcal{F}$ be a transposition of $i(N)$ and $j(N)$, i.e.

$$
\left\{\begin{array}{l}
\left(T_{1(N)} T_{1(N)} x\right)_{i(N)}=\left(T_{1(N)} x\right)_{j(N)}=x_{i(N)}, \text { and } \\
\left(T_{1(N)} x\right)_{K}=x_{K} \text { for all } K \in \mathbb{N} \backslash\{i(N), j(N)\}
\end{array}\right.
$$

By (17), P1 and P3, we have $\left(\boldsymbol{T}_{1(N)} \boldsymbol{P} \boldsymbol{x}\right)^{-M} \succ_{L}^{M}\left(\boldsymbol{T}_{1(N)} \boldsymbol{S} \boldsymbol{z}\right)^{-M}$ for all $M \in\{N \bar{m}+$ $1, \ldots, i(N)\}$. Moreover, by $\mathbf{P 2},\left(\boldsymbol{T}_{1(N)} \boldsymbol{P} \boldsymbol{x}\right)^{-(N+1) \bar{m}} \sim_{L}^{(N+1) \bar{m}}(\boldsymbol{P} \boldsymbol{x})^{-(N+1) \bar{m}}$, and $\left(\boldsymbol{T}_{1(N)} \boldsymbol{S} \boldsymbol{z}\right)^{-(N+1) \bar{m}} \sim_{L}^{(N+1) \bar{m}}(\boldsymbol{S} \boldsymbol{z})^{-(N+1) \bar{m}}$. Thus, by (15) and the transitivity of $\succsim_{L}^{(N+1) \bar{m}}$,

$$
\left(\boldsymbol{T}_{1(N)} \boldsymbol{P} \boldsymbol{x}\right)^{-(N+1) \bar{m}} \succ_{L}^{(N+1) \bar{m}}\left(\boldsymbol{T}_{1(N)} \boldsymbol{S} \boldsymbol{z}\right)^{-(N+1) \bar{m}} .
$$

Using the same argument repeatedly at most $t(N):=(N+1) \bar{m}-i(N)$ times (redefine $i(N)$ for the streams obtained after the transpositions for each case), we have, for all $M \in\{N \bar{m}+1, \ldots,(N+1) \bar{m}\}$,

$$
\left(\boldsymbol{T}_{t(N)} \ldots \boldsymbol{T}_{1(N)} \boldsymbol{P} \boldsymbol{x}\right)^{-M} \succ_{L}^{M}\left(\boldsymbol{T}_{t(N)} \ldots \boldsymbol{T}_{1(N)} \boldsymbol{S} \boldsymbol{z}\right)^{-M}
$$

Using the sequence of transpositions $\left\{\boldsymbol{T}_{1(1)}, \ldots, \boldsymbol{T}_{t(1)}, \boldsymbol{T}_{1(2)}, \ldots\right\}$, we define infinite- 
dimensional matrices $\tilde{\boldsymbol{P}}$ and $\tilde{\boldsymbol{S}}$ as: for all $N \in \mathbb{N}$,

$$
\left\{\begin{array}{l}
\tilde{\boldsymbol{P}}(N \bar{m})=\left[\boldsymbol{T}_{t(N)} \ldots \boldsymbol{T}_{1(2)} \boldsymbol{T}_{t(1)} \ldots \boldsymbol{T}_{1(1)} \boldsymbol{P}\right](N \bar{m}) \\
\tilde{\boldsymbol{S}}(N \bar{m})=\left[\boldsymbol{T}_{t(N)} \ldots \boldsymbol{T}_{1(2)} \boldsymbol{T}_{t(1)} \ldots \boldsymbol{T}_{1(1)} \boldsymbol{S}\right](N \bar{m}) .
\end{array}\right.
$$

By (18), $\tilde{\boldsymbol{P}}(N \bar{m})$ and $\tilde{\boldsymbol{S}}(N \bar{m})$ are well-defined as finite-dimensional permutation matrices for all $N \in \mathbb{N}$. Thus, $\tilde{\boldsymbol{P}}, \tilde{\boldsymbol{S}} \in \mathcal{Q}$. By (19) where $N \in \mathbb{N}$ is arbitrarily chosen, we obtain $(\tilde{\boldsymbol{P}} \boldsymbol{x})^{-m} \succ_{L}^{m}(\tilde{\boldsymbol{S}} \boldsymbol{z})^{-m}$ for all $m \geq \bar{m}$.

We now show that $\succsim_{Q L w}$ is a SWR on $X$.

(Reflexivity): Since $\boldsymbol{I} \in \mathcal{Q}$ and $\succsim_{L w}$ is reflexive, $\succsim_{Q L w}$ is also reflexive.

(Transitivity): By Lemma 2, $\succsim_{Q L w}$ is quasi-transitive. We show that $\boldsymbol{x} \succsim_{Q L w} \boldsymbol{z}$ follows for each of the three cases: (i) $\boldsymbol{x} \succ_{Q L w} \boldsymbol{y}$ and $\boldsymbol{y} \sim_{Q L w} \boldsymbol{z}$; (ii) $\boldsymbol{x} \sim_{Q L w} \boldsymbol{y}$ and $\boldsymbol{y} \succ_{Q L w} \boldsymbol{z}$; and (iii) $\boldsymbol{x} \sim_{Q L w} \boldsymbol{y}$ and $\boldsymbol{y} \sim_{Q L w} \boldsymbol{z}$. In case (i), by (3a) and (3b), there exist $\boldsymbol{P}, \boldsymbol{Q}, \boldsymbol{R} \in \mathcal{Q}$ such that $\boldsymbol{P} \boldsymbol{x} \succ_{L w} \boldsymbol{Q} \boldsymbol{y}$ and $\boldsymbol{R} \boldsymbol{y} \sim_{L w} \boldsymbol{z}$. Since $\boldsymbol{Q} \boldsymbol{R}^{\prime} \in \mathcal{Q}$, $(\boldsymbol{Q} \boldsymbol{y}=) \boldsymbol{Q} \boldsymbol{R}^{\prime} \boldsymbol{R} \boldsymbol{y} \sim_{L w} \boldsymbol{Q} \boldsymbol{R}^{\prime} \boldsymbol{z}$ follows from Lemma 1. The transitivity of $\succsim_{L w}$ gives $\boldsymbol{P} \boldsymbol{x} \succ_{L w} \boldsymbol{Q} \boldsymbol{R}^{\prime} \boldsymbol{z}$, and $\boldsymbol{x} \succ_{Q L w} \boldsymbol{z}$ follows from (3a). In the case of (ii), by (3a) and (3b), there exist $\boldsymbol{P}, \boldsymbol{Q}, \boldsymbol{R} \in \mathcal{Q}$ such that $\boldsymbol{P} \boldsymbol{x} \sim_{L w} \boldsymbol{y}$ and $\boldsymbol{Q} \boldsymbol{y} \succ_{L w} \boldsymbol{R} \boldsymbol{z}$. Since $\boldsymbol{Q P} \in \mathcal{Q}$, we can prove $\boldsymbol{x} \succ_{Q L w} \boldsymbol{z}$ by the similar argument to case (i), and we omit it. Finally, we consider case (iii). By (3b), there exist $\boldsymbol{P}, \boldsymbol{Q} \in \mathcal{Q}$ such that $\boldsymbol{P} \boldsymbol{x} \sim_{L w} \boldsymbol{y}$ and $\boldsymbol{Q} \boldsymbol{y} \sim_{L w} \boldsymbol{z}$. Since $\boldsymbol{Q P} \in \mathcal{Q}$, we obtain $\boldsymbol{x} \sim_{Q L w} \boldsymbol{z}$ by the similar argument to the case (i), and we omit it.

\section{C: Proofs of Theorems 1 and 2}

Proof of Theorem 1. The if part is obvious from the argument in Sect. 3. We provide the proof of the only if part. Assume that a SWR $\succsim$ satisfies SP, QA, WPC, and HE.

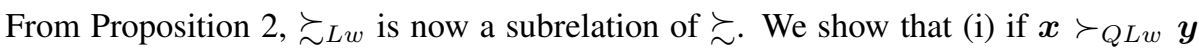
then $\boldsymbol{x} \succ \boldsymbol{y}$ and (ii) if $\boldsymbol{x} \sim_{Q L w} \boldsymbol{y}$ then $\boldsymbol{x} \sim \boldsymbol{y}$.

(i) Assume $x \succ_{Q L w} y$. By (3a), there exist $\boldsymbol{P}, \boldsymbol{Q} \in \mathcal{Q}$ such that $\boldsymbol{P} \boldsymbol{x} \succ_{L w} \boldsymbol{Q} \boldsymbol{y}$. Since $\succsim_{L w}$ is a subrelation of $\succsim, \boldsymbol{P} \boldsymbol{x} \succ \boldsymbol{Q} \boldsymbol{y}$. By QA, $\boldsymbol{x}=\boldsymbol{P}^{\prime} \boldsymbol{P} \boldsymbol{x} \sim \boldsymbol{P} \boldsymbol{x}$ and $\boldsymbol{y}=\boldsymbol{Q}^{\prime} \boldsymbol{Q} \boldsymbol{y} \sim \boldsymbol{Q} \boldsymbol{y}$. By the transitivity of $\succsim, \boldsymbol{x} \succ \boldsymbol{y}$.

(ii) Assume $\boldsymbol{x} \sim_{Q L w} \boldsymbol{y}$. By (3b), there exists $\boldsymbol{P} \in \mathcal{Q}$ such that $\boldsymbol{P} \boldsymbol{x} \sim_{L w} \boldsymbol{y}$. Since $\succsim_{L w}$ is a subrelation of $\succsim, \boldsymbol{P} \boldsymbol{x} \sim \boldsymbol{y}$. By $\mathbf{Q A}, \boldsymbol{x}=\boldsymbol{P}^{\prime} \boldsymbol{P} \boldsymbol{x} \sim \boldsymbol{P} \boldsymbol{x}$. Since $\succsim$ is transitive, $\boldsymbol{x} \sim \boldsymbol{y}$.

Proof of Theorem 2. Using Proposition 3, the same argument as in the proof of Theorem 1 can be applied to prove Theorem 2, and we omit it. 


\section{References}

Arrow, K.J.: Social Choice and Individual Values. Wiley, New York (1963)

Asheim, G.B., d'Aspremont, C., Banerjee, K.: Generalized time-invariant overtaking. Mimeo, University of Oslo (2008)

Asheim, G.B., Buchholz, W., Tungodden, B.: Justifying sustainability. J Environ Econ Manage 41, 252-268 (2001)

Asheim, G.B., Tungodden, B.: Resolving distributional conflicts between generations. Econ Theory 24, 221-230 (2004)

d'Aspremont, C.: Formal welfarism and intergenerational equity. In: Roemer, J., Suzumura, K. (eds.) Intergenerational Equity and Sustainability, pp. 113-130. Palgrave, London (2007)

d'Aspremont, C., Gevers, L.: Social welfare functionals and interpersonal comparability. In: Arrow, K.J., Sen, A.K., Suzumura, K. (eds.) Handbook of Social Choice and Welfare I, pp. 459-541. North-Holland, Amsterdam (2002)

Banerjee, K.: On the extension of the utilitarian and Suppes-Sen social welfare relations to infinite utility streams. Soc Choice Welf 27, 327-339 (2006)

Basu, K., Mitra, T.: Utilitarianism for infinite utility streams: a new welfare criterion and its axiomatic characterization. J Econ Theory 133, 350-373 (2007)

Blackorby, C., Bossert, W., Donaldson, D.: Utilitarianism and the theory of justice. In: Arrow, K.J., Sen, A.K., Suzumura, K. (eds.) Handbook of Social Choice and Welfare I, pp. 543-596. North-Holland, Amsterdam (2002)

Bossert, W., Sprumont, Y., Suzumura, K.: Ordering infinite utility streams. J Econ Theory 135, 579-589 (2007)

Bossert, W., Weymark, J.A.: Utility in social choice. In: Barbara, S., Hammond, P.J., Seidl, C. (eds.) Handbook of Utility Theory, Extensions, II, pp. 1099-1177. Kluwer, Boston (2004)

Brock, W.A.: An axiomatic basis for the Ramsey-Weizsäcker overtaking criterion. Econometrica 38, 927-929 (1970)

Fleurbaey, M., Michel, P.: Intertemporal equity and the extension of the Ramsey criterion. J Math Econ 39, 777-802 (2003)

Hammond, P.J.: Equity, Arrow's conditions, and Rawls' difference principle. Econometrica 44, 793-804 (1976) 
Kamaga, K., Kojima, T.: $\mathcal{Q}$-anonymous social welfare relations on infinite utility streams. Mimeo, Waseda University (2008)

Lauwers, L.: Rawlsian equity and generalised utilitarianism with and infinite population. Econ Theory 9, 143-150 (1997a)

Lauwers, L.: Infinite utility: insisting on strong monotonicity. Aust J Philos 75, 222233 (1997b)

Lauwers, L.: Ordering infinite utility streams: completeness at the cost of a nonRamsey set. Mimeo, Katholike Universiteit Leuven (2006)

Mitra, T., Basu, K.: On the existence of Paretian social welfare quasi-orderings for infinite utility streams with extended anonymity. In: Roemer, J., Suzumura, K. (eds.) Intergenerational Equity and Sustainability, pp. 85-99. Palgrave, London (2007)

Sakai, T.: Intergenerational equity and an explicit construction of welfare criteria. Mimeo, Yokohama National University (2008)

Sen, A.K.: Collective Choice and Social Welfare. Holden-Day, Amsterdam (1970)

Suppes, P.: Some formal models of grading principles. Synthese 6, 284-306 (1966)

Svensson, L.G.: Equity among generations. Econometrica 48, 1251-1256 (1980)

Szpilrajn, E.: Sur l'extension de l'ordre partiel. Fundam Math 16, 386-389 (1930)

van Liedekerke, L.: Should utilitarians be cautious about an infinite future. Aust J Philos 73, 405-407 (1995)

Zame, W.: Can intergenerational equity be operationalized? Theoret Econ 2: 187-202 (2007) 